\title{
Effects of Hydrodynamic Coupling on Electro-Optical Transients
}

\author{
Jan M. Antosiewicz ${ }^{\dagger}$ and Dietmar Porschke, ${ }^{*+}$ \\ Department of Biophysics, University of Warsaw, 02-089 Warsaw, Poland, Max Planck Institut für \\ biophysikalische Chemie, 37077 Göttingen, Germany, Tel. -551-2011438; Fax -551-2011168; \\ dpoersc@gwdg.de
}

Received: May 29, 2009; Revised Manuscript Received: July 16, 2009

\begin{abstract}
The effects resulting from coupling between translational and rotational diffusion on electro-optical transients were analyzed by Brownian dynamics simulations. Diffusion tensors, including translational-rotational coupling tensors, were derived from bead model simulations. Optical and electrical parameters were assigned according to models and experimental data. Hydrodynamic coupling has a strong impact on the shape of electro-optical transients for objects with a nonsymmetric structure. As expected, hydrodynamic coupling in general does not affect the time constants derived from decay curves. However, special attention is required at singular points, where amplitudes are inverted. Under these conditions, transients are observed with artificially reduced time constants, suggesting compact structures. False conclusions may be avoided, when experimental data are analyzed over a sufficiently wide range of conditions. Because transients induced upon reversal of the field vector are strongly affected by hydrodynamic coupling, dipole types cannot be assigned from these transients simply according to standard rules, when the shape of the objects is nonsymmetric. The time constants obtained from exponential fitting of rise-curves show unexpected dependencies on the field strength, which are mainly due to superposition of individual components with amplitudes of opposite sign that are not resolved upon fitting. Dipole parameters obtained from stationary degrees of orientation via orientation functions may also be strongly affected by hydrodynamic coupling. The high sensitivity of electro-optical transients on details of molecular shape and optical and electrical parameters provides a powerful approach to molecular analysis, but quantitative assignments require special care. When symmetry is lost, which must be expected because of bending in many cases, hydrodynamic coupling effects cannot be neglected.
\end{abstract}

\section{Introduction}

Electro-optical procedures ${ }^{1-3}$ proved to be very useful for the analysis of structures in solution. Macromolecules, colloids, and nanoparticles of various types have been characterized. The quantitative analysis of experimental data is very simple, provided that the shape of the particles can be approximated by simple geometric bodies like ellipsoids or rods. More efforts are required for particles with a complex shape, where model simulations are necessary for quantitative interpretations. A special case is given for particles with a nonsymmetric shape, because coupling between translational and rotational diffusion ${ }^{4,5}$ may lead to a considerable modification of electro-optical transients. ${ }^{6-10}$ In the past, hydrodynamic coupling has not been studied sufficiently well because of the fact that quantitative conclusions require rather extensive computer simulations. For extension of general information on the effects resulting from hydrodynamic coupling, we have simulated transients for simple model cases and analyzed these data with special emphasis on potential problems in their interpretation.

The program used for our simulations has been tested previously ${ }^{10}$ by comparison with analytical results available in the limit of low electric field strengths. We found complete agreement of the results obtained by the analytical and our Brownian dynamics approach.

Most of our present simulations are on a smoothly bent 179 bp DNA double helix as a simple model with a nonsymmetric

\footnotetext{
* Corresponding author. E-mail: dpoersc@gwdg.de.

$\uparrow$ University of Warsaw.

* Max Planck Institut für biophysikalische Chemie.
}

shape. For comparison, we have also simulated dichroism transients for a straight rod with the dimensions of a $182 \mathrm{bp}$ DNA double helix.

\section{Methods}

The Brownian dynamics simulations are based on the procedure developed by Ermak and McCammon. ${ }^{11}$ Details of our simulation program were described previously. ${ }^{10}$ Because of recent discussions on singularities ${ }^{12}$ in algorithms for Brownian dynamics simulations, we note that our algorithm is free of such singularities. The hydrodynamic, optical, and electrical parameters of our models were also obtained as described previously. ${ }^{3,13-15}$ The parameters of our smoothly bent $179 \mathrm{bp}$ DNA double helix include the following: total charge $(179 \times$ $2 \times \mathrm{e}) \times 0.12=6.883 \times 10^{-18} \mathrm{C}$ (the factor 0.12 results from ion condensation ${ }^{16}$ ); quasi-permanent dipole moment $\mu=8.96$ $\times 10^{-27} \mathrm{Cm}$ (in the direction of the $x$ axis); diagonal elements of the absorbance tensor $0.35507,0.4665$, and 0.17842 (cf. Figure 1 in ref 10); the $x$ and $z$ components of the polarizability 0.27893 and 0.72107 were approximated as described by Antosiewicz and Porschke ${ }^{13}$ and combined with the experimental value $3 \times 10^{-32} \mathrm{Cm}^{2} \mathrm{~V}^{-1}$ assumed to be valid for the straight DNA fragment; and diffusion tensor as given in eq 9 of ref 10 . The parameters of the straight rod corresponding to a $182 \mathrm{bp}$ DNA double helix include the following: diagonal elements of the absorbance tensor $0.4665,0.4665,0.06699$; quasi-permanent dipole 0 ; polarizability $3 \times 10^{-32} \mathrm{Cm}^{2} \mathrm{~V}^{-1}$; diagonal elements of the translational diffusion tensor $2.14826 \times 10^{-11} \mathrm{~m}^{2} \mathrm{~s}^{-1}$, $2.14826 \times 10^{-11} \mathrm{~m}^{2} \mathrm{~s}^{-1}, 3.2476 \times 10^{-11} \mathrm{~m}^{2} \mathrm{~s}^{-1}$; and of the 


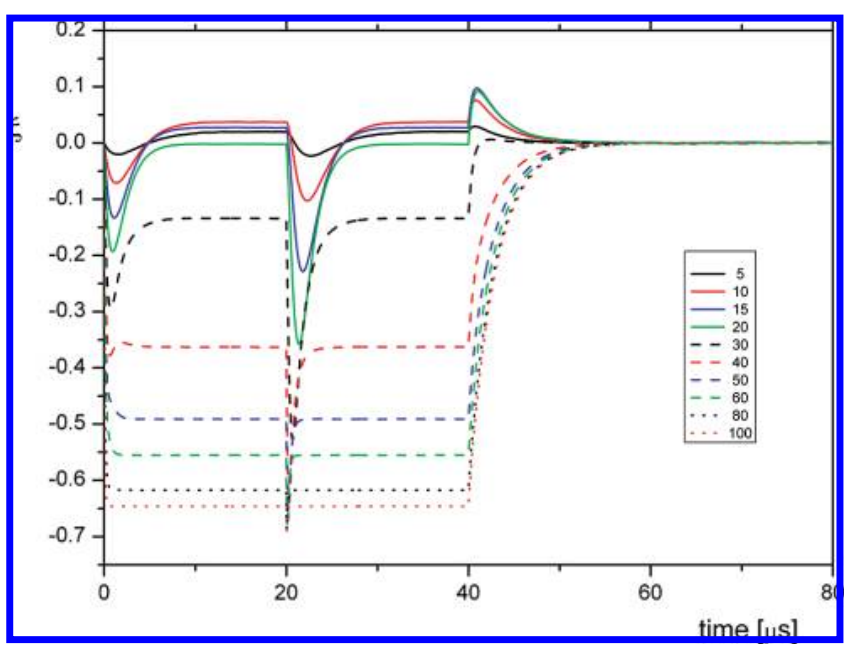

Figure 1. Reduced electric dichroism $\xi$ as a function of time $t$ for smoothly bent 179 bp DNA calculated with hydrodynamic coupling at different electric field strengths, given in $\mathrm{kV} / \mathrm{cm}$. The electric field pulse was applied at $t=0$ and terminated at $t=40 \mu \mathrm{s}$; the sign of the field vector was reverted at $t=20 \mu \mathrm{s}$.

rotational diffusion tensor $4.40998 \times 10^{-6} \mathrm{~s}^{-1}, 4.40998 \times 10^{-6}$ $\mathrm{s}^{-1}, 2.22182 \times 10^{-8} \mathrm{~s}^{-1}$.

All simulations were done at a temperature of $20{ }^{\circ} \mathrm{C}$. All of the transients given in this report are the average of at least $10^{6}$ single trajectories. In critical cases, simulations were extended to $7 \times 10^{6}$ single trajectories. Dichroism values ${ }^{1}$ are given in the form of the reduced dichroism $\xi=\left(\Delta \epsilon_{\|}-\Delta \epsilon_{\perp}\right) / \epsilon$.

For our calculations, we used the facilities of the Gesellschaft für wissenschaftliche Datenverarbeitung $\mathrm{mbH}$, Göttingen.

\section{Results}

Smoothly Bent 179 bp DNA. The simple model used for most of our simulations is a smoothly bent rod with hydrodynamic, optical, and electrical parameters corresponding to those of a DNA fragment with 179 base pairs. The contour length of such fragment is close to the persistence length of DNA double helices. ${ }^{17}$ The bending angle of our model was selected to get the same end-to-end distance as the root-mean-square end-toend distance of a worm like chain with a contour length of 179 base pairs and a persistence length of $450 \AA$.

Stationary Dichroism. Sample sets of transients calculated with and without hydrodynamic coupling are shown in Figures 1 and 2, respectively. The stationary values were described previously but were not analyzed quantitatively. Stationary values measured at different electric field strengths are commonly used ${ }^{1,3}$ to determine dipole parameters. In the present case, we have analyzed the Brownian dynamics data by an orientation function for a model with an induced dipole in one direction and a permanent dipole moment in a perpendicular direction to the induced dipole. As shown in Figure 3, the stationary values obtained by simulations with hydrodynamic coupling can be fitted at a reasonable accuracy by this orientation function. The polarizability obtained from least-squares fitting is close to the expected value, whereas the fitted permanent dipole moment is higher by a factor of $\sim 3.5$ than that used in the Brownian dynamics simulations. Thus, hydrodynamic coupling may lead to a considerable deviation of fitted parameters. For a control, we have simulated 10 stationary dichroism values in the range from 5 to $100 \mathrm{kV}$ for a model with a polarizability of $2.16 \times 10^{-32} \mathrm{Cm}^{2} \mathrm{~V}^{-1}$ along the long axis and a permanent dipole of $8.96 \times 10^{-27} \mathrm{Cm}$ perpendicular to the

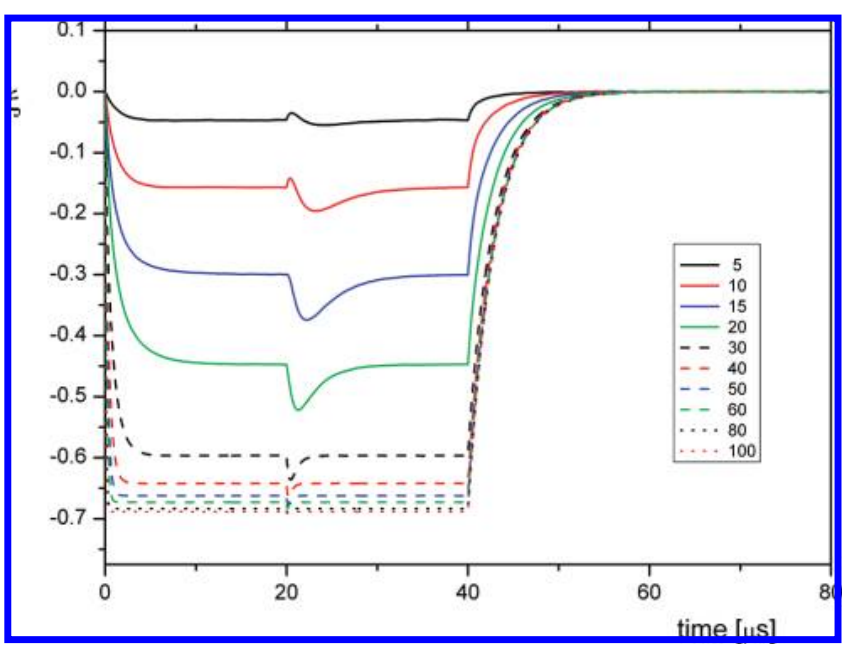

Figure 2. Reduced electric dichroism $\xi$ as a function of time $t$ for smoothly bent $179 \mathrm{bp}$ DNA calculated without hydrodynamic coupling at different electric field strengths, given in $\mathrm{kV} / \mathrm{cm}$. The electric field pulse was applied at $t=0$ and terminated at $t=40 \mu \mathrm{s}$; the sign of the field vector was reverted at $t=20 \mu \mathrm{s}$.

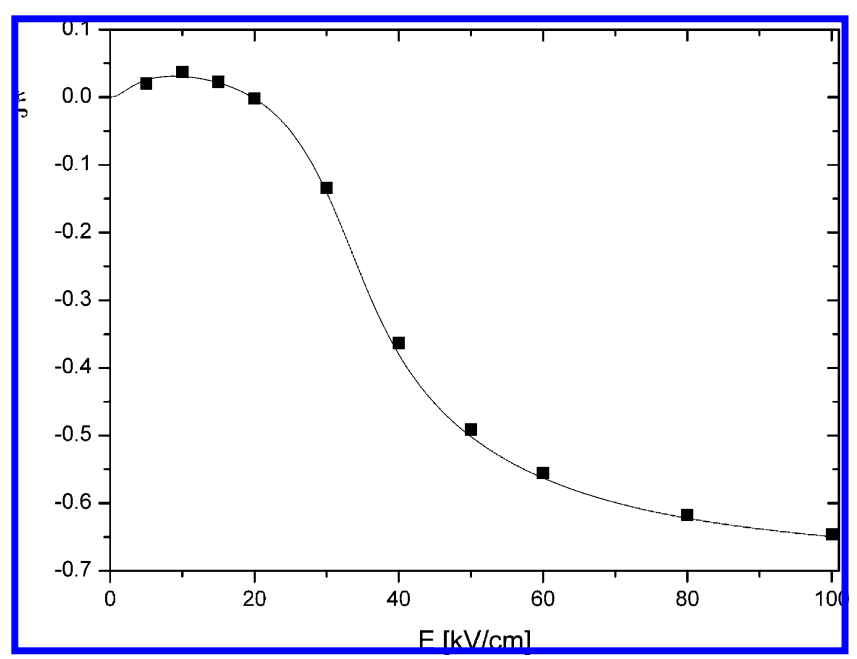

Figure 3. Stationary dichroism $\xi$ as a function of the electric field strength $E$. The squares represent values obtained from Brownian dynamics simulations for the smoothly bent 179 bp DNA (parameters as in Methods) with hydrodynamic coupling. The continuous line represents a least-squares fit of these values to an orientation function for a particle with an induced and a permanent dipole in perpendicular direction to each other. Fitted parameters: $p=1.48 \times 10^{-32} \mathrm{Cm}^{2} \mathrm{~V}^{-1}$; $\mu=3.40 \times 10^{-26} \mathrm{Cm}$; the components of the extinction tensor used in the Brownian dynamics simulation were applied in the fit procedure. The simulation parameters included an $\mathrm{x}$-component of the polarizability (see also Methods). When this component was turned to 0 and stationary dichroism data were simulated, a fit of these data provided $p=3.27 \times$ $10^{-32} \mathrm{Cm}^{2} \mathrm{~V}^{-1}$ and $\mu=4.77 \times 10^{-26} \mathrm{Cm}$; thus, the deviation was even higher.

long axis in the absence of hydrodynamic coupling. Fitting of these data to the orientation function used above provided the expected parameters, within limits of accuracy $\pm 2 \%$.

Pulse Reversal. Another important source of information is the transients induced by reversal of the field vector. A simple comparison of the transients given in Figures 1 and 2 shows a considerable effect of hydrodynamic coupling on their shape. The shape of these transients has been recommended as a particularly reliable indicator for the contribution of permanent versus induced dipole moments. ${ }^{1,18}$ Application of the simple rules given in the literature for such assignments leads to erroneous conclusions, when the objects are nonsymmetric. It 


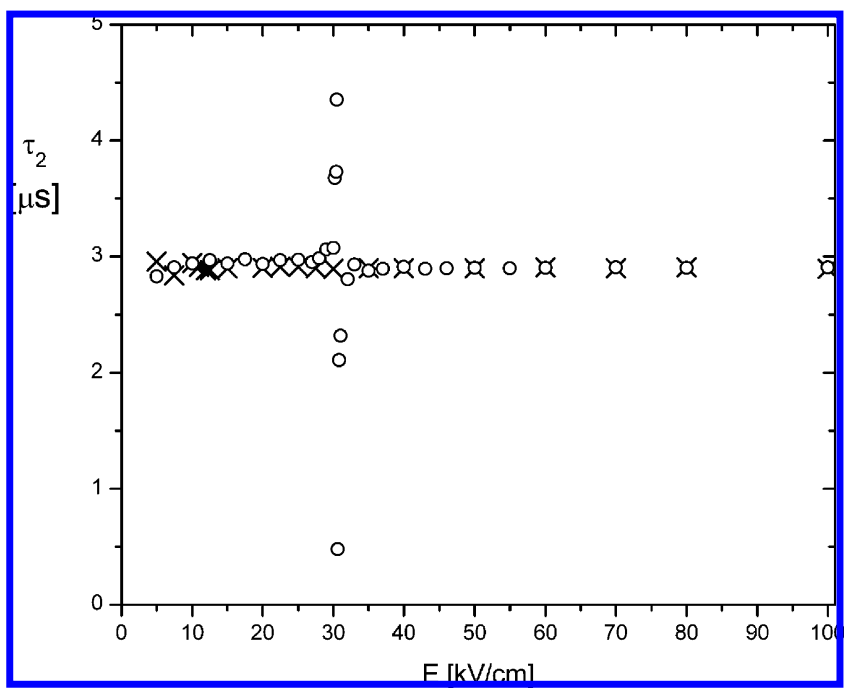

Figure 4. Exponential time constants $\tau_{2}$ fitted to dichroism decay curves for the smoothly bent 179 bp DNA as a function of the electric field strength $E$ (applied before pulse termination) in the presence (o) and in the absence $(x)$ of hydrodynamic coupling.

may be possible to construct rules for compensation of errors. However, such rules would be rather complex and therefore not sufficiently useful in practice.

Decay-Curves. Important information on the size, shape, and flexibility of the objects under investigation is derived from the decay curves. We have analyzed the simulated decay curves by least-squares fitting programs, in close analogy to standard experimental investigations with particular emphasis on the slowest exponential component. In most cases, we get the expected "slow" time constant of $2.9 \mu$ s (Figure 4). However, there is a special range of the electric field strength where the time constants show remarkable deviations. The deviations appear when the amplitude of the slow decay component changes its sign. This is demonstrated in Figure 5 for a decay curve simulated at the electric field strength of $30.6 \mathrm{kV} / \mathrm{cm}$. The fit with the lowest sum of squared deviations indicates two exponentials with a "slow" component of $0.478 \mu$ s comprising almost all of the amplitude. The simulated data have very little noise, and the deviation of the fit from these data may hardly be recognized. An indication for something special in these data is provided by the autocorrelation of the deviations (see Figure 5). Under standard experimental conditions with the usual noise level, it is virtually impossible to recognize anything particular in such data. This remains a problem for data simulated in the environment of the inversion point, which is simply due to the fact that in this range the slow component is associated with very low relative amplitude. Thus, the reason for the apparent acceleration effect is quite obvious.

We do not have any simple explanation for another effect observed in the close environment of the inversion point, where the time constants obtained by least-squares fitting are larger than the expected value (see Figure 4). This is remarkable because the fitted values are larger than the largest exponential component predicted for our model by the equations of Wegener, Dowben, and Koester. ${ }^{19}$ The amplitude associated with the large fitted time constants is relatively small, in the range of $\sim 1 \%$ of the total amplitude. Although these effects were observed repeatedly, an artifact cannot be excluded yet.

The decay curves simulated in the absence of hydrodynamic coupling do not show any special features. Least squares fits with 2 exponentials are satisfactory in the range of electric fields



Figure 5. Exponential fit of a dichroism decay curve simulated for the smoothly bent $179 \mathrm{bp}$ DNA at $30.6 \mathrm{kV} / \mathrm{cm}$. The simulated data cannot be distinguished from the fit with two exponentials $\tau_{1}=3.3 \mathrm{~ns}$, $\tau_{2}=0.48 \mu \mathrm{s}\left(\mathrm{A}_{1}=-0.0049, \mathrm{~A}_{2}=0.152\right)$. The lower panel shows the difference between the simulated and the fitted data; the inset shows the autocorrelation of the deviations.

strengths from 5 to $100 \mathrm{kV} / \mathrm{cm}$. The average time constant obtained for the slow process is $2.903 \mu$ s with a standard deviation of $0.014 \mu \mathrm{s}$. The deviations observed at low electric field strengths (see Figure 4) are relatively large because of the low dichroism amplitude. A systematic dependence on the field strength has not been detected.

A close look at the data given in Figure 4 shows that the $\tau_{2}$ values obtained with hydrodynamic coupling in the range 40 to $100 \mathrm{kV}$ are identical $\left(\tau_{2}=2.903 \pm 0.005 \mu \mathrm{s}\right)$ to those obtained without hydrodynamic coupling in the full range of field strengths. However, a difference is observed in the range from 10 to $25 \mathrm{kV}$, where the average $\tau_{2}$ value is $2.957 \pm 0.018$ $\mu \mathrm{s}$ in the case with hydrodynamic coupling. This difference is relatively small but is out of the noise level of the present simulations.

Rise-Curves. Most of the rise-curves can be fitted by two exponentials at a reasonable accuracy. In this case, a dependence on the electric field strength is expected, whereas the details revealed by our simulations have not been anticipated. It should be emphasized that theory predicts in general eight exponential contributions for rise-curves at low field strengths. ${ }^{6}$ Thus, results from fits by two exponentials may be misleading. However, our approach is analogous to that of experimentalists, who determine the minimum number of exponentials required for reasonable fits. In the absence of hydrodynamic coupling, the time constant for the slow process increases with the field strength in the range from 10 to $15 \mathrm{kV} / \mathrm{cm}$, before a decrease follows in the range above $15 \mathrm{kV} / \mathrm{cm}$ (see Figure 6). The data with hydrodynamic coupling show a more complex dependence on the field strength: a decrease of $\tau_{2}$ in the range from 5 to $17.5 \mathrm{kV} / \mathrm{cm}$ is followed by an increase from 17.5 to $27 \mathrm{kV} / \mathrm{cm}$; then, another decrease is observed before a discontinuity seems to appear between 40 and $43 \mathrm{kV} / \mathrm{cm}$; finally, $\tau_{2}$ decreases at field strengths above $43 \mathrm{kV} / \mathrm{cm}$. A closer look at the range with the "discontinuity" demonstrates that the decay curves in this 




Figure 6. Exponential time constants $\tau_{2}$ fitted to dichroism rise curves for the smoothly bent $179 \mathrm{bp}$ DNA as a function of the electric field strength $E$ in the presence (o) and in the absence $(\times)$ of hydrodynamic coupling. The three data points marked by $(\diamond)$ refer to cases where deviations of the simulated data at the given low noise level from a two exponential fit are clearly visible (see also text and Figure 7).

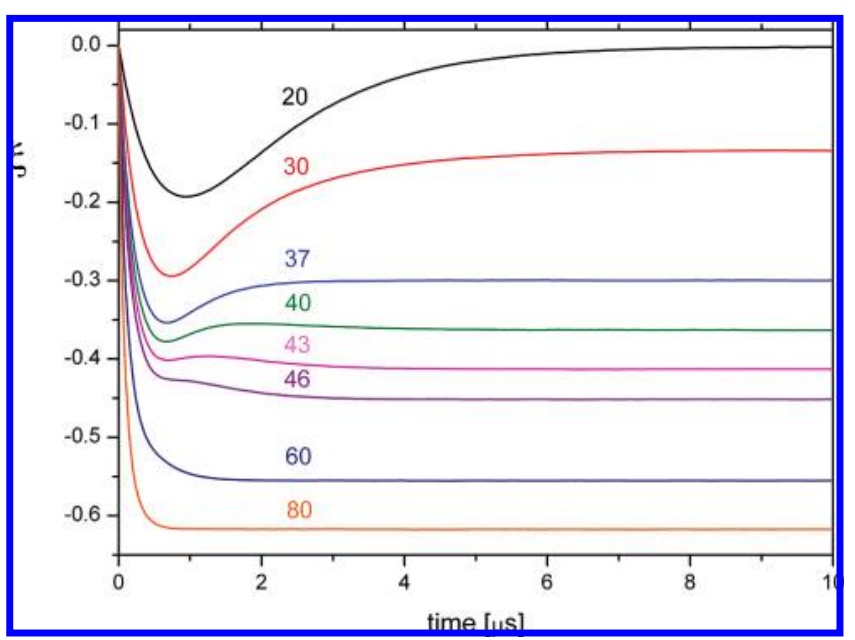

Figure 7. Dichroism rise-curves for the smoothly bent 179 bp DNA at different electric field strengths. The numbers represent the respective electric field strengths in $\mathrm{kV} / \mathrm{cm}$.

range require three exponentials for accurate fitting (see Figure 7). Thus, the "discontinuity" is not real, but experimental data with noise may suggest the existence of such unusual phenomenon. Again, the special effect appears in a range where the sign of the amplitude for a relaxation component is inverted.

Although not always clearly visible, this type of effect seems to be the main reason for unexpected dependences on the electric field strength. Another example (not shown) is the rise-curve simulated without hydrodynamic coupling at $5 \mathrm{kV} / \mathrm{cm}$, where a "slow" component with small amplitude opposite to that of the main effect appears. This component is not clearly visible anymore at higher field strengths but contributes by superposition with other components to an "apparent acceleration" of the rise-curves.

Straight Rod. For comparison, we have simulated dichroism transients for a straight rigid rod with dimensions corresponding to a 182 bp DNA double helix. In this case, there is no coupling between translational and rotational diffusion. The decay curves can be fitted at a high accuracy by single exponentials over the full range of field strengths up to $100 \mathrm{kV} / \mathrm{cm}$. The time constants fitted in the range of electric field strengths from 5 to $100 \mathrm{kV} /$ $\mathrm{cm}$ are $3.784 \pm 0.003 \mu$ s and do not show any dependence on the field strength within the limits of accuracy.

The rise-curves can also be fitted by single exponentials. The risetime constants obtained from these fits decrease with increasing field strength and are consistent with an equation given by Schwarz ${ }^{20}$

$$
1 / \tau=\frac{2 p D_{r}}{k T x_{0}} E^{2}
$$

The parameters are $p$ polarizability, $\mathrm{D}_{\mathrm{r}}$ rotational diffusion coefficient, $E$ electric field strength, $k T$ thermal energy, and $x$ a factor which is approximately 3.35. Linear regression of the data obtained in the range from 1 to $100 \mathrm{kV} / \mathrm{cm}$ provided a factor $x=3.08$.

In a previous simulation ${ }^{7}$ for a DNA with $84 \mathrm{bp}$ and polarizabilities 1.67 and $5.8 \times 10^{-33} \mathrm{Cm}^{2} \mathrm{~V}^{-1}$, the factors $x$ were 2.86 and 2.93 , respectively. Thus, all of the data simulated for the straight DNA with 182 bp are consistent with expectation.

\section{Discussion}

As shown before ${ }^{6-10}$ hydrodynamic coupling between translational and rotational movements may affect electro-optical transients extensively, provided that the particles under investigation are nonsymmetric. The magnitude of this effect depends on various parameters including the size, the charge, and the type of asymmetry. The special effect on the orientation of the particles detected by birefringence or electric dichroism measurements is induced by the rapid translational motion of the particles under external electric fields. Because of this electrophoretic motion, the different orientations of nonsymmetric particles in space are not equivalent anymore. The orientation due to electrophoretic motion is superimposed on the orientation of the particles resulting from induced and/or permanent dipoles. Thus, electrophoretic motion affects both transients and stationary states.

Our calculations of the hydrodynamic coupling effect are based on the tensor description of translation and rotational diffusion, which proved to be very successful in the representation of experimental data for various macromolecules. ${ }^{3,15,21} \mathrm{We}$ have shown previously ${ }^{10}$ that the implementation in our Brownian dynamics algorithm provides the same results as the analytical approach in the limit of low electric field strengths. Thus, all reasonable and currently possible controls have been executed.

In the present investigation, we have continued our analysis of the effect of hydrodynamic coupling on electro-optical transients. Some general conclusions have been described previously. ${ }^{10}$ Here, we prepared a more detailed analysis with particular emphasis on the time constants and with consideration of reversed pulses.

Electro-optical transients induced by reversal of the field vector $^{1,18}$ have been recommended as a particularly reliable approach for the characterization of electrical dipoles. The present results demonstrate that this approach may be quite misleading, when the overall shape of the particles under investigation is not symmetric. Particular attention is required for large nonsymmetric objects, where the effects of hydrodynamic coupling cannot be neglected. Because there are various phenomena affecting these transients in addition to hydrodynamic coupling, it is not practicable anymore to present simple rules for interpretation of experimental data. Each case must 
be analyzed separately with consideration of various factors including hydrodynamic coupling.

Another problem resulting from hydrodynamic coupling is the extensive change of the stationary dichroism in a wide range of electric field strengths. As shown in Figure 3, interpretation of such data by standard orientation functions leads to erroneous dipole parameters. Correction of these dipole parameters is not trivial. The only practicable approach seems to be development of models, simulation of experimental data based on these models, and a careful comparison of simulations with experiments.

A very serious misinterpretation of experimental data may result when the analysis of decay curves is restricted to the limited range of electric field strengths, where inversion of the amplitude for the slow decay component occurs. The example given in Figure 5 demonstrates a dramatic apparent acceleration effect. If this effect is not assigned properly, then conclusions on the dimensions of particles under investigation will be completely wrong. Under experimental conditions with the usual noise level, the problem is not restricted to a singular value of the electric field strength but may extend over a range of field strengths. However, careful experimentalists may avoid trouble easily by taking measurements over a sufficiently wide range of electric field strengths.

The unusual dependences of the risetime constants on the electric field strength observed for the bend rod seem to be mainly due to superposition of many individual relaxation effects, which are not resolved upon fitting. According to theory, there are up to eight contributions, whereas fitting procedures often resolve only two relaxation processes, even at a relatively low noise level.

The magnitude of the effects induced by hydrodynamic coupling is strongly dependent on both the shape and the charge pattern of the particle under investigation. In the present case of a DNA double helix, we have used charge parameters indicated by polyelectrolyte theory. ${ }^{16}$ However, these charge parameters depend on the details of the ionic environment and may also be dependent to some degree on the local DNA structure, which is determined by the base sequence. Thus, variations in the electro-optical response observed for different DNA fragments may be due to this type of ion binding effect. Differences in ion binding are expected to be more evident at high concentrations.

A first experimental report ${ }^{22}$ of special effects corresponding to those observed in our current simulations has been presented for a DNA fragment with 179 base pairs in a buffer containing $11 \mathrm{mM} \mathrm{NaCl}$ and $1 \mathrm{mM}$ cacodylate $\mathrm{pH} 7.0$ at $2{ }^{\circ} \mathrm{C}$. At low field strengths, the electric dichroism decayed continuously as usual with a spectrum of two time constants $\tau_{1}=0.22 \mu$ s and $\tau_{2}=4.3 \mu \mathrm{s}$. The slower process has been assigned as usual to overall rotational motion. When the field strength was increased, the shape of the decay curves was changed such that the decay could be fitted by a single exponential with $\tau=0.57 \mu$ s. Upon a further increase of the field strength, bimodal decay curves appeared having a positive dichroism component with a slow decay and a negative dichroism component with a fast decay. These experimental results are very similar to the simulated ones and suggest that the model used in our present simulations describes the properties of the DNA with 179 base pairs exactly.

The agreement is remarkable in view of the fact that the model used in our simulations was rigid, whereas DNA double helices are considered to be flexible. We also accept that DNA double helices are flexible, but the time scale of this flexibility remains to be established. It is possible that there are slow modes of bending converting different bending states of DNA to each other with a spectrum of time constants slower than that of the dichroism decay. In this case, our present simulations should describe the experiments correctly.

For a final conclusion on the time scale of DNA bending flexibility, simulations are required on flexible chains. Actually, there are some simulations on flexible chains, ${ }^{21,23-26}$ but the details of these simulations do not correspond closely enough to those of the electro-optical experiments on DNA double helices.

\section{Conclusions}

Special attention is required in the analysis of electro-optical data obtained for nonsymmetric objects because hydrodynamic coupling may cause serious misinterpretations. However, problems, for example, caused by "discontinuities", may be avoided by careful analysis of data measured in a sufficiently wide range of conditions.

Acknowledgment. The orientation function for a particle with an induced and a permanent dipole moment in perpendicular orientation to each other was constructed by Dr. Max Teubner (MPI Biophys. Chem.). His contribution is acknowledged gratefully.

\section{References and Notes}

(1) Fredericq, E.; Houssier, C. Electric dichroism and electric birefringence; Clarendon Press: Oxford, 1973.

(2) Stoylov, S. P. Colloid electro-optics: theory, techniques, applications; Academic Press: London, 1991.

(3) Porschke, D.; Antosiewicz, J. M. Quantitative molecular electrooptics: Macromolecular structures and their dynamics in solution. In Molecular and colloidal electro-optics; Stoylov, S. P., Stoimenova, M. V., Eds.; CRC: Boca Raton, FL, 2007; pp 55.

(4) Brenner, H. J. Colloid Sci. 1965, 20, 104.

(5) Brenner, H. J. Colloid Interface Sci. 1967, 23, 407.

(6) Wegener, W. A. J. Chem. Phvs. 1986, 84, 5989.

(7) Antosiewicz, J.; Grycuk, T.; Porschke, D. J. Chem. Phys. 1991, 95, 1354

(8) Bertolotto, J.; Roston, G.; Ascheri, M.; Campo, M. Phvsica a-Statistical Mechanics and Its Applications 2003, 327, 185.

(9) Bertolotto, J. A.; Roston, G. B.; Ascheri, M. E. Prog. Colloid Polvm. Sci. 2004, 128, 25 .

(10) Porschke, D.; Antosiewicz, J. M. J. Phvs. Chem. B 2005, 109, 1034. (11) Ermak, D. L.; McCammon, J. A. J. Chem. Phvs. 1978, 69, 1352.

(12) Evensen, T. R.; Vaess, S. V.; Elgsaeter, A. Macromol. Theory Simul. 2009, 18, 50 .

(13) Antosiewicz, J.; Porschke, D. J. Biomol. Struct. Dvn. 1988, 5, 819.

(14) Antosiewicz, J.; Porschke, D. J. Phvs. Chem. 1989, 93, 5301.

(15) Garcia De La Torre, J.; Navarro, S.; Lopez Martinez, M. C.; Diaz,

F. G.; Lopez Cascales, J. J. Biophvs. J. 1994, 67, 530.

(16) Manning, G. S. Q. Rev. Biophvs. 1978, 11, 179.

(17) Porschke, D. Biophvs. Chem. 1991, 40, 169.

(18) Tinoco, I.; Yamaoka, K. J. Phvs. Chem. 1959, 63, 423.

(19) Wegener, W. A.; Dowben, R. M.; Koester, V. J. J. Chem. Phvs

1979, 70,622 .

(20) Schwarz, G. Zeitschrift Fur Phvsik 1956, 145, 563.

(21) Garcia de la Torre, J. Colloid. Surf. B-Biointerfaces 2007, 56, 4.

(22) Antosiewicz, J.; Porschke, D. Biophvs. Chem. 1989, 33, 19.

(23) Navarro, S.; Lopez Martinez, M. C.; Garcia de la Torre, J. J. Chem. Phys. 1995, 103, 7631.

(24) Diaz, F. G.; Carrasco, B.; Lopez Martinez, M. C.; Garcia de la Torre, J. J. Phys. Chem. B 2000, 104, 12339.

(25) Elvingson, C. Biophvs. Chem. 1992, 43, 9.

(26) Elvingson, C. Chem. Phys. Lett. 1993, 214, 91.

JP9050403 